\title{
Transcranial Doppler sonography during head up tilt suggests preserved central sympathetic activation in familial dysautonomia
}

\author{
M J Hilz, F B Axelrod, U Haertl, C M Brown, B Stemper
}

J Neurol Neurosurg Psychiatry 2002;72:657-660

\begin{abstract}
Objective: Cerebral autoregulation was assessed by transcranial Doppler sonography in 10 patients with familial dysautonomia and 10 age matched controls.

Methods: Blood pressure, heart rate, and middle cerebral artery blood flow velocity (CBFV) were simultaneously recorded when supine and during 180 seconds of head up tilt. Cerebrovascular resistance (CVR) was calculated from CBFV and mean blood pressure was adjusted to brain level.

Results: In the controls, mean blood pressure remained stable during tilt, but heart rate increased significantly. In the patients with familial dysautonomia, mean (SD) blood pressure decreased by $15.0(10.8) \%(p<0.05)$. Heart rate remained unchanged. In controls, systolic and mean CBFV decreased by 9.1 (4.7)\% and 9.4 (7.0)\%, respectively, while diastolic CBFV remained stable. In the patients, diastolic and mean CBFV decreased continuously by 32.1 (13.9)\% and by 14.8 (31.4)\%. Supine CVR was $28 \%$ higher in patients than in controls and decreased significantly less during head up tilt.

Conclusions: Tilt evokes orthostatic hypotension without compensatory tachycardia in patients with familial dysautonomia owing to decreased peripheral sympathetic innervation. High supine CVR values and relatively preserved CVR during tilt suggest preserved central sympathetic activation in familial dysautonomia, assuring adaptation of cerebrovascular autoregulation to chronic supine hypertension and orthostatic hypotension.
\end{abstract}

$\mathrm{F}$ amilial dysautonomia is an autosomal recessive disorder with extensive deficit of sensory, sympathetic, and some parasympathetic neurones. ${ }^{1}$ Peripheral sympathetic failure is considered to be the cause of cardiovascular lability. ${ }^{1-3}$ However, impaired cerebrovascular autoregulation might contribute to autonomic dysregulation, with orthostatic hypotension and other disturbance such as cardiac arrhythmias, syncope at rest, and sudden death.

To investigate central autonomic dysfunction in patients with familial dysautonomia, we assessed cerebral vascular perfusion and cerebral autoregulation during passive head up tilt. $^{45}$

\section{METHODS}

Ten patients with familial dysautonomia (five women and five men, mean (SD) age 26.9 (9.4) years) and 10 healthy controls (four women and six men, age 25.2 (12.7) years) participated in the study. All the patients with familial dysautonomia fulfilled accepted diagnostic criteria ${ }^{1}$ and had the characteristic genetic haplotype. ${ }^{6}$ Only patients who were able to discontinue their drug treatment (fludrocortisone or midodrine) 18 hours before testing participated in the study. Informed consent was obtained according to the declaration of Helsinki.

An $80^{\circ}$ head up tilt was initiated after 25 minutes of supine rest. Cerebrovascular and cardiovascular responses were analysed during three minutes of tilt. We monitored heart rate and systolic, diastolic, and mean blood pressure $\left(\mathrm{BP}_{\text {sys' }}, \mathrm{BP}_{\text {dia }}\right.$ $\mathrm{BP}_{\text {mean }}$ ) using left radial artery applanation tonometry with the wrist at heart level, ${ }^{7}$ end tidal $\mathrm{CO}_{2}\left(\right.$ Colin Pilot $^{\mathrm{TM}}{ }^{\mathrm{T}}$, San Antonio, Texas, USA), respiratory frequency using inductance plethysmography (Respitrace ${ }^{\mathrm{TM}}$, Ardsley, New York, USA), and systolic, diastolic, and mean cerebral blood flow velocities $\left(\mathrm{CBFV}_{\mathrm{sy}}\right.$, $\mathrm{CBFV}_{\text {dia }^{\prime}}\left(\mathrm{CBFV}_{\text {mean }}\right)$ of the left or right middle cerebral artery using transtemporal Doppler sonography (35-55 mm insonation depth, $2 \mathrm{MHz}$ probe; Pioneer ${ }^{\mathrm{TM}}$, EME/Nicolet, Madison, Wisconsin, USA). ${ }^{8}$

Cerebrovascular resistance (CVR) was calculated as mean blood pressure adjusted to brain level $\left(\mathrm{BP}_{\text {mean_brain }}\right)$ divided by $\mathrm{CBFV}_{\text {mean }}$. We calculated $\mathrm{BP}_{\text {mean brain }}$ as the difference between $\mathrm{BP}_{\text {mean }}$ and the hydrostatic pressure effect $\left(\mathrm{BP}_{\text {hydro }}\right)$ at the level of transcranial insonation, as described elsewhere. ${ }^{10}$ All participants were instructed to maintain their regular breathing pattern during the test.

Biosignals were sampled synchronously at $1 \mathrm{kHz}$ and transferred after analogue to digital conversion into a data acquisition and analysis system (HRview ${ }^{\mathrm{TM}}, \mathrm{BMT}^{\mathrm{TM}}$, Wakefield, Massachusetts, USA).

Average baseline values were obtained during a 60 second interval that ended 30 seconds before tilting to avoid any bias by activities preceding the tilt. For each second of tilt, variables were determined in relation to baseline values. ${ }^{7}$

Sympathetic and parasympathetic heart rate modulation was assessed from 256 second epochs at baseline and during tilt, as previously described. ${ }^{7}$ Low frequency heart rate oscillations (LF: $0.04-0.15 \mathrm{~Hz}$ ) are mediated by sympathetic and parasympathetic activity at rest ${ }^{11}{ }^{12}$ and predominantly by sympathetic activity during tilt ${ }^{12}$; high frequency oscillations (HF: 0.04-0.15 Hz) reflect parasympathetic activity. ${ }^{11}{ }^{12}$

From the heart rate tachogram at baseline and during head up tilt we calculated the root mean square successive differences (RMSSD) as a measure of parasympathetic activity, ${ }^{11}$ and the standard deviation (SD) and coefficient of variation (CV), both reflecting sympathetic and parasympathetic heart rate modulation. ${ }^{11}$

\section{Statistics}

Data are presented as mean (SD). Mean values during the 180 second tilt were compared with baseline values by a two sided

\footnotetext{
Abbreviations: $\mathrm{BP}_{\text {mean brain, }}$ blood pressure adjusted to brain level; $\mathrm{CBFV}$, cerebral blood flow velocity; CVR, cerebrovascular resistance; HF, high frequency; LF, low frequency; RMSSD, root mean square of successive
} differences; TCD, transcranial Doppler 
Table 1 Cardiovascular and cerebrovascular variables in controls and patients with familial dysautonomia in the supine position and during 180 seconds of head up tilt

\begin{tabular}{|c|c|c|c|c|c|c|c|c|}
\hline \multirow{3}{*}{$\begin{array}{l}\text { Variable } \\
\text { Heart rate (beats/min) }\end{array}$} & \multicolumn{4}{|c|}{ Controls } & \multicolumn{4}{|c|}{ FD patients } \\
\hline & \multicolumn{2}{|l|}{ At rest } & \multicolumn{2}{|c|}{ During HUT } & \multicolumn{2}{|l|}{ At rest } & \multicolumn{2}{|c|}{ During HUT } \\
\hline & 68.0 & (9.9) & 84.4 & (14.6) & 77.0 & $(9.1)$ & 78.4 & $(8.9)$ \\
\hline $\mathrm{BP}_{\text {sys }}(\mathrm{mm} \mathrm{Hg})$ & 104.9 & (8.2) & 105.2 & (18.1) & 138.2 & $(22.8)$ & 119.0 & (15.4) \\
\hline $\mathrm{BP}_{\text {mean }}(\mathrm{mm} \mathrm{Hg})$ & 72.3 & $(7.6)$ & 77.0 & (17.2) & 101.1 & (19.3) & 84.5 & $(10.2)$ \\
\hline $\mathrm{BP}_{\text {dia }}(\mathrm{mm} \mathrm{Hg})$ & 56.0 & (8.1) & 62.9 & (13.9) & 82.5 & (18.1) & 67.2 & $(8.6)$ \\
\hline $\mathrm{CBVF}_{\text {sys }}(\mathrm{cm} / \mathrm{s})$ & 95.5 & (13.9) & 86.4 & (10.9) & 94.6 & (23.8) & 93.4 & $(24.3)$ \\
\hline $\mathrm{CBFV}_{\text {mean }}(\mathrm{cm} / \mathrm{s})$ & 65.4 & (8.9) & 58.6 & (8.3) & 71.1 & $(18.4)$ & 54.5 & (14.2) \\
\hline $\mathrm{CBFV}_{\text {dia }}(\mathrm{cm} / \mathrm{s})$ & 46.1 & $(5.9)$ & 43.5 & $(6.6)$ & 54.0 & (10.4) & 37.4 & $(9.4)$ \\
\hline CVR (mm Hg.s. $\left.\mathrm{cm}^{-1}\right)$ & 1.14 & $(0.2)$ & 0.84 & $(0.2)$ & 1.46 & (0.37) & 1.19 & (0.33) \\
\hline Respiratory frequency (cycles/min) & 20.3 & $(5.0)$ & 16.6 & $(5.8)$ & 22.6 & $(4.8)$ & 22.8 & $(6.4)$ \\
\hline End tidal $\mathrm{CO}_{2}(\mathrm{~mm} \mathrm{Hg})$ & 39.6 & (4.3) & 39.0 & (3.8) & 41.0 & (4.8) & 40.2 & (3.4) \\
\hline RMSSD & 73.3 & (42.2) & 24.8 & (15.0) & 28.7 & (14.4) & 19.9 & $(4.4)$ \\
\hline $\mathrm{CV}$ & 8.1 & $(2.9)$ & 6.4 & $(1.7)$ & 5.4 & $(2.3)$ & 4.8 & (1.7) \\
\hline SD & 0.072 & $2(0.023)$ & 0.04 & $6(0.05)$ & 0.042 & $2(0.019)$ & 0.035 & $5(0.009)$ \\
\hline LF ([beats/min $]^{2}$ ) & 7.1 & (4.3) & 16.3 & (11.1) & 4.4 & (3.8) & 4.8 & (6.0) \\
\hline $\mathrm{HF}\left([\text { beats } / \mathrm{min}]^{2}\right)$ & 13.1 & (17.3) & 5.4 & (7.3) & 2.4 & (2.3) & 1.8 & (1.3) \\
\hline
\end{tabular}

Values are mean (SD).

Bold numbers indicate significant differences between supine and head up tilt values (Wilcoxon: $p<0.05$ ).

$\mathrm{BP}_{\text {dia, }}$ diastolic blood pressure; $\mathrm{BP}_{\text {mean }}$ mean blood pressure; $\mathrm{BP}_{\text {sys }}$ systolic blood pressure; $\mathrm{CBVF}$, cerebral

blood flow velocity; $\mathrm{CVR}$, cerebral vascular resistance, calculated as ratio of $\mathrm{BP}_{\text {mean }}$ (adjusted to brain level)

to $\mathrm{CBFV}_{\text {mean }}$ HF, high frequency power of heart rate oscillations $(0.04-0.15 \mathrm{~Hz}) ; \mathrm{HUT}$, head up tilt; LF, low

frequency power of heart rate oscillations $(0.04-0.15 \mathrm{~Hz})$; RMSSD, root mean square of successive

differences.

Wilcoxon test. Differences between patient and control values were evaluated by the $\mathrm{U}$ test. The level of significance was set at $\mathrm{p} \leqslant 0.05$ (SYSTAT, Evanston, Illinois, USA).

\section{RESULTS}

Table 1 shows the supine values of the patient and control groups. When supine, heart rate and blood pressure values were higher in the patients than in the controls. CBFV, respiratory frequency, and end tidal $\mathrm{CO}_{2}$ did not differ between the groups. CVR was $28 \%$ higher in the patients than in the controls, but the difference was not statistically significant.

Upon tilt, the heart rate of the controls increased immediately (average increase, 23.9 (8.8)\%) (fig 1). Blood pressure remained stable, with only $\mathrm{BP}_{\text {dia }}$ increasing (by 14.2 (28.3)\%). Systolic, mean, and diastolic CBFV values decreased slightly but not significantly in controls.

In the patients, blood pressure showed a pronounced decrease upon tilt. After 20 seconds, the blood pressure increased intermittently for about 20 seconds and then steadily declined. After 80 seconds of head up tilt, $\mathrm{BP}_{\mathrm{sys}}$ had already fallen by $15.0(12.9) \%, \mathrm{BP}_{\text {mean }}$ by $21.4(12.3) \%$, and $\mathrm{BP}_{\text {dia }}$ by 15.8 (12.6)\%. Diastolic and mean CBFV decreased immediately upon tilt. Within 15 seconds, $\mathrm{CBFV}_{\text {dia }}$ and $\mathrm{CBFV}_{\text {mean }}$ were significantly reduced (fig 1). While both velocities continued to decline, $\mathrm{CBFV}_{\text {sys }}$ started to recover after 20-30 seconds of tilt and regained baseline velocities.

The decrease was greater for $\mathrm{CBFV}_{\text {dia }}$ and $\mathrm{CBFV}_{\text {mean }}$ and smaller for $\mathrm{CBFV}_{\mathrm{sys}}$ in the patients than in the controls. During head up tilt, CVR decreased less in the patients (18.5\%) than in the controls $(26.3 \%)$.

Respiratory frequency remained stable and end tidal $\mathrm{CO}_{2}$ decreased slightly but not significantly in both groups during tilt (table 1).

Heart rate variability showed higher supine RMSSD and SD values in the controls than in the patients (table 1). During tilt, RMSSD and SD decreased significantly in controls but not in the patients. Spectral powers of heart rate modulation in the LF and HF range were lower in the patients than in the controls (table 1). During tilt, HF power decreased and LF power increased in the controls; in the patients, neither LF nor HF power differed from the supine condition, but the values were significantly lower than in the controls.

\section{DISCUSSION}

During tilt, our controls had normal cardiovascular responses, with parasympathetic withdrawal and sympathetic activation as expressed by increased LF power and decreased RMSSD, CV, and HF power of heart rate. ${ }^{11}{ }^{12}$ Cerebral autoregulation was also normal and assured almost unchanged cerebral blood flow values. ${ }^{8}{ }^{13}$ In both groups, end tidal $\mathrm{CO}_{2}$ changes were minimal and would not explain any changes in cerebral blood flow velocity. ${ }^{14}$

The patients with familial dysautonomia showed impaired peripheral cardiovascular and cerebrovascular responses during tilt. The peripheral sympathetic deficiency was evidenced by postural hypotension without compensatory tachycardia ${ }^{13}$ and can be attributed to a reduction in sympathetic neurones in the intermediolateral column and the sympathetic ganglia ${ }^{1}$ as well as to an absence of autonomic vascular nerve terminals. ${ }^{15}$ However, the intermediate blood pressure recovery after the onset of tilt suggests an incomplete peripheral denervation, allowing for some residual peripheral vasoconstriction (fig 1). The inadequate decrease of RMSSD, SD, and HF power probably reflects additional cardiovagal dysfunction-for example, the baroreflex failure that occurs in familial dysautonomia ${ }^{3}$ and impairment of central cardiovagal activation, which has been noted independently of baroreflex function. ${ }^{7}$

In the patients with familial dysautonomia, mean and diastolic cerebral blood flow velocity decreased while systolic velocity increased during tilt. Theoretically, this discrepancy might be the result of a temporary improvement in cardiac performance caused by a reduction in afterload on tilting. In a patient with diabetic autonomic neuropathy, Novak et al observed impaired cerebral autoregulation similar to our findings, with a decrease in mean and diastolic cerebral blood flow velocity and stable systolic velocity during orthostatic hypotension..$^{5}$ These investigators attributed the preserved systolic flow to an increase in cardiac contractility during orthostasis. ${ }^{5}$ However, such a mechanism should stabilise systolic blood flow immediately on tilt. In our patients, systolic blood flow velocity only returned to baseline values after 20 to 30 seconds. It is more likely that the decrease in diastolic flow with maintenance of systolic flow during tilting result from a change in downstream perfusion of the small cerebral resistance vessels. ${ }^{16}$ 

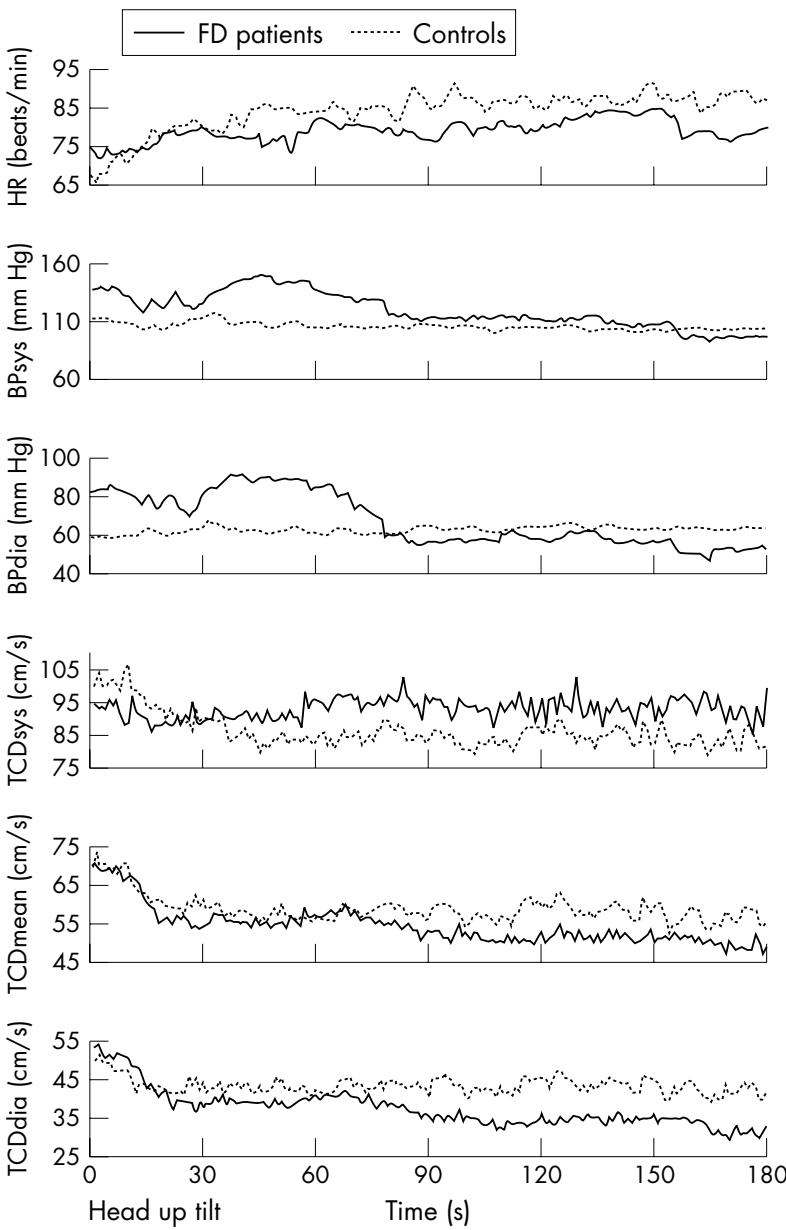

Figure 1 Averaged values of heart rate (HR), systolic and diastolic blood pressure (BPsys and BPdia), and systolic, mean, and diastolic cerebral blood flow velocities (CBFV), determined by transcranial Doppler (TCDsys, TCDmean, and TCDdia) in 10 patients with familial dysautonomia (FD) and 10 controls during head up tilt. In the controls, blood pressure remained stable. Heart rate increased during tilt in the controls, but did not change in the patients. In the patients, blood pressure initially decreased over 20 seconds, with a brief recovery lasting another 20 seconds, followed by a secondary steady decline. In the controls, there was a mild decrease in CBFV, but overall $C B F V$ remain stable. In the patients, all CBFV values initially decreased, but then only systolic CBFV increased.

Both Schondorf et al and Grubb et al observed a similar decrease in diastolic cerebral blood flow velocity with unchanged systolic velocity during orthostatic hypotension in patients with neurally mediated syncope. ${ }^{13}{ }^{17}$ Schondorf et al attributed the selective slowing of diastolic velocity with unchanged systolic velocity to a passive collapse of small vessels and a sudden orthostatic fall in systemic blood pressure, the driving force of cerebral perfusion. ${ }^{13}$ These investigators assume that neurally mediated syncope results from an excessive dilatation and consequent passive collapse of small vessels occurring when arterial critical closing pressure exceeds venous pressure. ${ }^{13}$ Our measurements of cerebral vascular resistance suggest that this mechanism of exaggerated dilatation is unlikely to be operative in the patients with familial dysautonomia. In the supine position, vascular resistance was approximately $28 \%$ higher in our patients than in the controls, which assured a similar blood flow velocity despite the supine hypertension in the patients. During head up tilt, cerebral vascular resistance decreased less in the patients than in the controls. These findings indicate that the tone of intracerebral resistance vessels is higher in the patients, not only during supine hypertension but also during progressive orthostatic hypotension. Therefore, a passive collapse of excessively dilated vessels during tilt-as suggested by Schondorf et al ${ }^{13}$-does not seem to explain the changes in cerebral vascular resistance and blood flow velocity.

The changes in these variables in our patients are more readily explained by the mechanisms discussed by Grubb et al. ${ }^{17}$ Those investigators attributed the decrease in diastolic cerebral blood flow velocity with a stable systolic velocity to "paradoxic intracerebral vasoconstriction" during increasing hypotension. Owing to their deficient peripheral sympathetic nervous system, patients with familial dysautonomia cannot maintain peripheral vasoconstriction and blood pressure. However, they seem able to generate a degree of tone in the intracerebral resistance vessels sufficient to counterregulate supine hypertension and prevent syncope despite hypotension. We conclude that there is a discrepancy between the peripheral sympathetic deficiency and a preserved ability to activate central sympathetic or neuropeptide outflow, both maintaining cerebral vessel tone in the face of supine hypertension or orthostatic hypotension. ${ }^{4}{ }^{18}$

In the controls, the pronounced vasodilatation during head up tilt reflects normal autoregulation involving a reduction in sympathetic nerve activity and a decreased release of vasoconstricting chemical mediators and neuropeptides. ${ }^{48}$ In patients with familial dysautonomia, the mechanisms adapting cerebral autoregulation to chronic orthostatic hypotension are unknown but may involve increased central sympathetic outflow or neuropeptide release similar to the mechanisms postulated by Grubb et al for patients with neurally mediated syncope. ${ }^{17}$

\section{ACKNOWLEDGEMENT}

This study was partially funded by the Dysautonomia Foundation, New York, USA, and by Sanofi-Synthelabo Pharmaceuticals, Germany. BS was supported as a postdoctoral fellow by the research society "Deutsche Akademie der Naturforscher Leopoldina", Germany.

\section{Authors' affiliations}

F B Axelrod, B Stemper, Department of Neurology, New York University, New York, USA

M J Hilz, U Haertl, C M Brown, Department of Neurology, University of Erlangen-Nuremberg, Erlangen, Germany

Correspondence to: Dr Max J Hilz, Department of Neurology, University of Erlangen-Nuremberg, Schwabachanlage 6, 91054 Erlangen,

Germany; max.hilz@neuro.med.uni-erlangen.de

Received 7 June 2001

In revised form 14 December 2001

Accepted 18 December 2001

\section{REFERENCES}

1 Axelrod FB, Hilz M. Familial dysautonomia. In: Vinken PJ, Bruyn GW, eds. Handbook of clinical neurology, vol 75. Amsterdam: Elsevier, 2000: 143-60.

2 Hilz MJ, Kolodny EH, Neuner I, et al. Highly abnormal thermotests in familial dysautonomia suggest increased cardiac autonomic risk. J Neurol Neurosurg Psychiatry 1998;65:338-43

3 Maayan C, Axelrod FB, Akselrod S, et al. Evaluation of autonomic dysfunction in familial dysautonomia by power spectral analysis. J Auton Nerv Syst 1987:21:51-8.

4 Hilz M, Stemper B, Heckmann J, et al. Mechanisms of cerebral autoregulation, assessment and interpretation by means of transcrania Doppler sonography. Fortschr Neurol Psychiatr 2000;68:398-412.

5 Novak P, Novak V, Low PA, et al. Transcranial Doppler evaluation in disorders of reduced orthostatic tolerance. In: Low PA, ed. Clinical autonomic disorders. Philadelphia: Lippincott-Raven, 1997:349-68.

6 Slaugenhaupt SA, Blumenfeld A, Gill SP, et al. Tissue-specific expression of a splicing mutation in the IKBKAP gene causes familial dysautonomia. Am J Hum Genet 2001;68:598-605.

7 Hilz MJ, Stemper B, Sauer P, et al. Cold face test demonstrates parasympathetic cardiac dysfunction in familial dysautonomia. Am J Physiol 1999;276:R1833-9.

8 Aaslid R, Lindegaard KF, Sorteberg W, et al. Cerebral autoregulation dynamics in humans. Stroke 1989;20:45-52.

9 Burton AC. Physiology and biophysics of the circulation. Chicago: Year Book Medical Publishers, 1966. 
10 Kawai Y, Murthy G, Watenpaugh DE, et al. Cerebral blood flow velocity in humans exposed to $24 \mathrm{~h}$ of head-down tilt. J Appl Physiol 1993;74:3046-51

11 Task Force. European Society of Cardiology and the North American Society of Pacing and Electrophysiology. Heart rate variability: standards of measurement, physiological interpretation, and clinical use. Circulation 1996;93:1043-65

12 Goldstein B, Woolf PD, DeKing D, et al. Heart rate power spectrum and plasma catecholamine levels after postural change and cold pressor test. Pediatr Res 1994;36:358-63.

13 Schondorf R, Benoit J, Wein T. Cerebrovascular and cardiovascular measurements during neurally mediated syncope induced by head-up tilt. Stroke 1997;28:1564-8.
14 Blaber AP, Bondar RL, Stein F, et al. Transfer function analysis of cerebral autoregulation dynamics in autonomic failure patients. Stroke 1997; 28: 1686-92

15 Grover-Johnson N, Pearson J. Deficient vascular innervation in familial dysautonomia, an explanation for vasomotor instability. Neuropathol Appl Neurobiol 1976;2:217-24.

16 Reutern G, Buedingen H. Ultrasound diagnosis of cerebrovascular diseases. New York: Louis R Caplan, 1993

17 Grubb BP, Gerard G, Roush K, et al. Cerebral vasoconstriction during head-upright tilt-induced vasovagal syncope. A paradoxic and unexpected response. Circulation 1991;84:1157-64.

18 Ursino M. Mechanisms of cerebral blood flow regulation. Crit Rev Biomed Eng 1991;18:255-88 